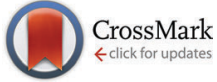

Cite this: Phys. Chem. Chem. Phys., $2016,18,16404$

Received 7th April 2016 Accepted 30th May 2016 DOI: $10.1039 / c 6 c p 02312 f$

www.rsc.org/pccp

\section{Cooperative enhancement versus additivity of two-photon-absorption cross sections in linear and branched squaraine superchromophores $\dagger$}

\author{
Harald Ceymann, ${ }^{a}$ Arnulf Rosspeintner, ${ }^{b}$ Maximilian H. Schreck, ${ }^{a}$ Carina Mützel, ${ }^{a}$ \\ Andreas Stoy, ${ }^{a}$ Eric Vauthey*b ${ }^{\star b} d$ Christoph Lambert ${ }^{\star a}$
}

\begin{abstract}
The linear and nonlinear optical properties of a series of oligomeric squaraine dyes were investigated by one-photon absorption spectroscopy (1PA) and two-photon absorption (2PA) induced fluorescence spectroscopy. The superchromophores are based on two indolenine squaraine dyes with transoid (SQA) and cisoid configuration (SQB). Using these monomers, linear dimers and trimers as well as star-shaped trimers and hexamers with benzene or triphenylamine cores were synthesised and investigated. The redshifted and intensified 1PA spectra of all superchromophores could well be explained by exciton coupling theory. In the linear chromophore arrangements we also found superradiance of fluorescence but not in the branched systems. Furthermore, the 2PA showed enhanced cross sections for the linear oligomers but only additivity for the branched systems. This emphasizes that the enhancement of the 2PA cross section in the linear arrangements is probably caused by orbital interactions of higher excited configurations.
\end{abstract}

\section{Introduction}

In recent years large two-photon-absorption (2PA) cross sections $\left(\delta_{2 \mathrm{PA}}\right)$ for quadrupolar-type $\mathrm{D}-\mathrm{A}-\mathrm{D}$ (or similarly in $\mathrm{A}-\mathrm{D}-\mathrm{A}$, where $\mathrm{A}$ is an electron acceptor and $\mathrm{D}$ is an electron donor) chromophores have been reported. ${ }^{1,2}$ The various applications of $2 \mathrm{PA}$, e.g. twophoton fluorescence spectroscopy, ${ }^{3,4} 3 \mathrm{D}$ microfabrication and optical data storage ${ }^{5}$ as well as photo dynamic therapy ${ }^{6,7}$ strongly benefit from high $\delta_{2 \mathrm{PA}}$ values. Furthermore the long wavelengths used in 2PA processes of D-A-D chromophores result in reduced scattering and an improved penetration of the medium, because of the absence of significant linear absorption in the $2 \mathrm{PA}$ region.

The magnitude of the cross section $\delta_{2 \mathrm{PA}}$ in symmetric systems can be estimated by eqn (1), with $\Gamma_{02}$ being the homogeneous linewidth of the 2PA transition, using a three-level model as depicted in Fig. 1.8,9

$$
\delta_{2 \mathrm{PA}}\left(E_{\mathrm{p}}\right) \propto \frac{E_{\mathrm{p}}^{2}}{\left(E_{01}-E_{\mathrm{p}}\right)^{2}+\Gamma_{01}{ }^{2}} \frac{\mu_{01}^{2} \mu_{12}^{2} \Gamma_{02}}{\left(E_{02}-2 E_{\mathrm{p}}\right)^{2}+\Gamma_{02}{ }^{2}}
$$

\footnotetext{
${ }^{a}$ Institut für Organische Chemie, Universität Würzburg, Wilhelm Conrad Röntgen Research Center for Complex Material Systems, Center for Nanosystems Chemistry, Am Hubland, D-97074 Würzburg, Germany. E-mail: christoph.lambert@uni-wuerzburg.de

${ }^{b}$ Department of Physical Chemistry, University of Geneva, 30 Quai Ernest-Ansermet, CH-1211 Geneva 4, Switzerland

$\dagger$ Electronic supplementary information (ESI) available: Synthetic protocols, fluorescence excitation anisotropy spectrum, power dependence of 2PAF, lifetime distributions and TD-DFT calculations. See DOI: 10.1039/c6cp02312f
}

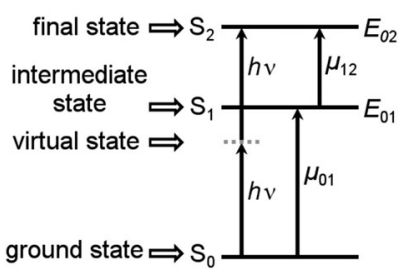

Fig. 1 Schematic representation of the 2PA process in the case of the three level model for symmetric systems.

From this simple model, it is obvious that in cases where the laser energy $E_{\mathrm{p}}$ is half the transition energy $E_{02}$ from the ground state to the 2PA state and at the same time is equal to the 1PA allowed transition $E_{01}$ a so called "double resonance" effect occurs which enhances the 2PA cross section dramatically..$^{8,10}$ In this respect squaraine dyes are particularly attractive chromophores. Their high 2PA cross section is attributed to a symmetric charge transfer from the donor (D) ends (=heterocyclic group) towards the acceptor (A = squaric acid moiety) of these D-A-D molecules, which increases the transition dipoles of 1PA and 2PA allowed states. Squaraines behave essentially as neutral cyanine dyes. As such, the 1PA to $S_{1}$ is spectrally very narrow. This allows approaching the $S_{1}$ state with $E_{\mathrm{p}}$ and using the "double resonance" situation to increase the 2PA cross section.

Calculations and experiments have shown that in substituted quadrupolar chromophores the donor end groups have a great influence on the transition moment between the $S_{1}$ and $S_{2}$ state $\mu_{12}$ while the extent of conjugation and the strength of the 
centred acceptor are correlated to $\mu_{01} \cdot{ }^{11,12}$ Webster et al. varied the strength of the acceptor bridge between two identical heterocyclic donor groups by using a polymethine dye, a squaraine dye and a tetraone core. They interpreted the large $\delta_{2 \mathrm{PA}}(8200 \mathrm{GM})$ of the squaraine dye being due to the increase of the density of final states and, thus, to the number of possible electronic transitions between the 1PA state and a state with twice that energy. In addition a large intermediate state resonance enhancement was observed due to the sharply rising low-energy side of the linear absorption spectra. ${ }^{13}$ These authors also used a squaraine chromophore as the bridge between two zinc porphyrin moieties, leading to very broad 2PA spectra with cross sections of up to $11000 \mathrm{GM}$ over the region of 1580 to $850 \mathrm{~nm} .{ }^{14}$ Moreshead et al. achieved a high 2PA cross section in molecules that consisted of two squaraines that were bridged by a fluorene moiety. ${ }^{15}$

Another way to accomplish large $2 \mathrm{PA}$ responses is the coupling of several chromophores. The ratio between the 2PA cross section $\delta_{2 \mathrm{PA}}^{(n)}$ of a superchromophore consisting of $n$ monomers and the 2PA cross section $\delta_{2 \mathrm{PA}}^{(1)}$ of the monomer is defined as $F_{(n)}$ in eqn (2). In the literature additive enhancement $\left(F_{(n)}=n\right)$, cooperative behaviour $\left(F_{(n)}>n\right)$ and weakening $\left(F_{(n)}<n\right)$ of the cross sections were reported. ${ }^{16,17}$ More specific, $\delta_{2 \mathrm{PA}}^{(n)}$ should scale with $n^{2}$ if full cooperativity is observed. ${ }^{18,19}$ This is caused by the linear dependence of both $\mu_{01}{ }^{2}$ and $\mu_{12}{ }^{2}$ on the number of chromophores in eqn (1). ${ }^{20}$

$$
F_{(n)}=\frac{\delta_{2 \mathrm{PA}}^{(n)}}{\delta_{2 \mathrm{PA}}^{(1)}}
$$

E.g. Blanchard-Desce et al. observed an enhancement effect for branched chromophores based on substituted triarylamines (TAA) but no such effect for star-like systems with a 1,3,5-substituted benzene (ben) core. These authors suggested coherent interactions mediated by wavefunction overlaps to be the source of the 2PA enhancement in case of triarylamines. ${ }^{17,21-23}$ Prasad et al. ${ }^{24}$ and Rebane et al. ${ }^{20}$ observed a similar effect in triarylamine based dendrimers. These authors also pointed out that one should not compare cross sections measured at only one wavelength because of spectral shifts in the chromophore aggregates.

In an older work Scherer et al. together with one of $\mathrm{us}^{25}$ observed an enhancement of the 2PA cross section in a series of thiophene-bridged oligosquaraines (up to pentamers). There, it was concluded that the low-lying 2PA allowed states are the result of conjugational effects which cannot be explained by purely electrostatic exciton coupling. The latter however deliver a reasonable explanation for the shifts of 1PA states.

In this work we again focus on the 2PA properties of superchromophores built up from squaraine dyes (see Fig. 2). ${ }^{26-34}$ Besides linear arrangements we also investigate branched systems with a triarylamine core or a benzene core. In addition to the wellinvestigated trans-squaraine parent chromophore $\mathbf{S Q A} \mathbf{A}^{9,14,15,25,35,36}$ we also employ the cis-squaraine dye SQB. In the latter system one oxygen atom of the central squaric acid is replaced by a dicyanovinylene group leading to higher acceptor strength and, as a result, to a red shift of the 1PA. In the homodimers ISQA and $\mathbf{d S Q B}$ and the homotrimers $\mathbf{T S Q A}$ and $\mathbf{T S Q B}$ we investigate the effect of direct coupling of dye monomers to yield oligomers on their 2PA properties. In the star-shaped compounds SQA-TAA, SQB-TAA, SQA-ben and dSQA-ben the branching effect is examined. We expect that in all oligomeric superchromophores, the nonlinear optical properties will change as the linear optical properties do because of exciton coupling effects. Thus, it is the goal of this work to examine whether exciton coupling of excited monomer states can be used to increase the 2PA of superchromophores. However, for all the above mentioned dyes one has to take into account that diverse conformers (rotational isomers via the biaryl axis) may be present in solution and the symmetry of compounds might be lower as indicated in Fig. 2 .

\section{Results and discussion}

\section{Absorption spectra}

Before we present the results of the 2PA measurements we will first briefly discuss the linear absorption spectra of the monomer dyes and their oligomers. This discussion will be based on the concept of exciton coupling, i.e. the coupling of transition moments of individual chromophores in order to produce the lowest energy excited states of the superchromophores. ${ }^{37-39}$ In Fig. 3 the 1PA spectra of the investigated squaraines in toluene are presented. The symmetrical monomer SQA (Fig. 3a) shows the typical absorption of a squaraine compound, i.e. one sharp and very intense band in the red region of the visible spectrum, originating from the HOMO $\rightarrow$ LUMO transition, and a weak shoulder on the high energy side which stems from vibronic progression.

For a dimer of SQA (=dSQA) with a head-to-tail orientation we may consider two idealised structures, a bent structure and a more stretched structure both with $C_{2}$ symmetry. Both structures can be formed by torsion around the biaryl axis. In Fig. 4, the exciton coupling interaction of localised transition moments (blue arrows) with their phase relation (direction of the arrows) for both scenarios is depicted. This theory predicts two possible electronic transitions into two exciton states which are separated by twice the exciton coupling energy $J$. While for the bent case both transitions are allowed, only the lowest exciton state is an allowed transition in case of the linear structure. In practice, mixtures of at least these two conformers will be present in solution and we expect to observe the lowest exciton state (termed $S_{1}$ in the following) as a more intense transition and the upper exciton state (termed $S_{1}{ }^{\prime}$ because it derives from the $\mathrm{S}_{1}$ state of the monomer) as a less intense peak (shoulder). Fig. 3a shows that for dSQA the more intense maximum at $14500 \mathrm{~cm}^{-1}$ is by $1000 \mathrm{~cm}^{-1}$ bathochromically shifted in comparison to SQA, and the weaker peak has an energy of $16100 \mathrm{~cm}^{-1}$. The difference of these two absorption maxima can be used to estimate the exciton coupling energy $J$ which is $c a .800 \mathrm{~cm}^{-1}$ (see Table 1).

For a head-to-tail arrangement of three chromophores as in tSQA we disregard different conformers completely and give only the qualitative solution of exciton coupling theory for a zig-zag arrangement. This is sketched in Fig. 4. Thus, exciton theory 

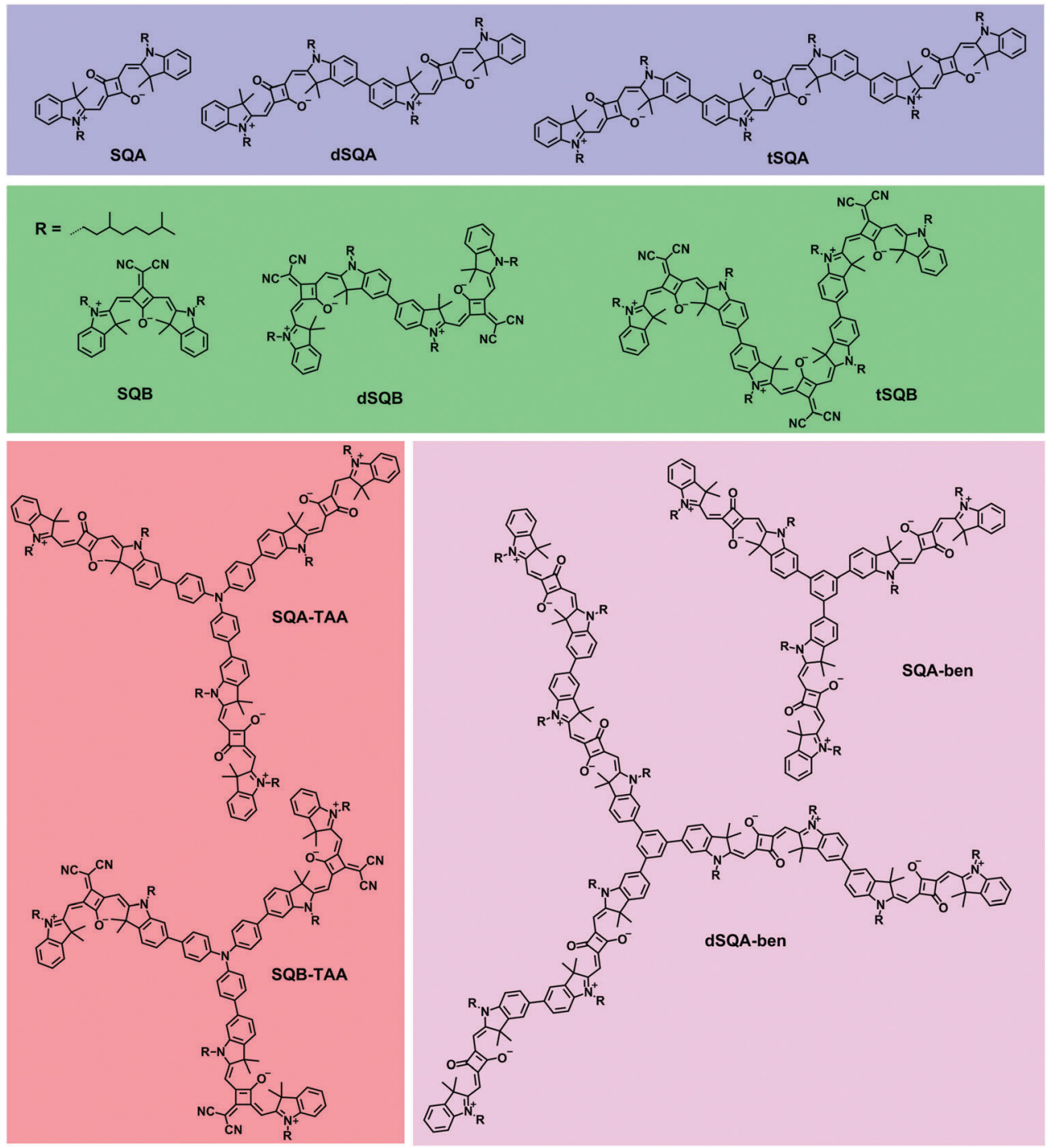

Fig. 2 Linear and star-shaped squaraine oligomers and their monomeric reference compounds.

predicts three exciton states where the lowest one is highly allowed, the highest one is slightly allowed but the intermediate state is forbidden. The energy splitting of the upper and lower exciton state is $2 \sqrt{2} J$. Again, in practice we expect that these selection rules are lifted because of the presence of different conformers. Indeed, for the trimer tSQA Fig. 3a shows three peak maxima with decreasing intensity from lower to higher energy. From the energy difference of the lowest $\left(14000 \mathrm{~cm}^{-1}\right)$ and the highest energy peak $\left(16100 \mathrm{~cm}^{-1}\right)$ we estimate an exciton coupling energy of $c a .740 \mathrm{~cm}^{-1}$ in good agreement with that of the dimer ISQA.

In the superchromophores derived from SQB the substitution of one oxygen atom by a dicyanomethylene group in the central squaric acid moiety leads to increased electron acceptor strength and a lowering of the LUMO energy. This shifts the lowest energy absorption by $c a .1100 \mathrm{~cm}^{-1}$ to the red compared to their SQA analogues (Table 1). The absorption spectra of the dimer dSQB and the trimer $\mathbf{T S Q B}$ are similar to their SQA analogues but show some variation in peak intensities (Fig. 3b).
The star-shaped trimers SQA-TAA and SQB-TAA with a triarylamine as the connecting core unit essentially show a broadening of the lowest energy absorption band which is indicative of a weak exciton coupling effect (Fig. 3c). The red shift of the absorption relative to their monomers is primarily due to the addition of the propeller-like triarylamine donor to the quadrupole dyes, thus breaking the $C_{2}$ symmetry of the squaraine moieties and inducing some CT character. ${ }^{40,41}$ In contrast the trimer SQA-ben with benzene as the connecting bridge displays a small shoulder at $c a .15700 \mathrm{~cm}^{-1}$ on the high energy side of the main absorption band which could be caused by a weak exciton coupling (Fig. 3d). This transition is forbidden for a truly $C_{3}$ symmetric chromophore as indicated in Fig. 4 and is only visible because of some structural disorder.

A similar shoulder is observed for the larger dSQA-ben. Here, it is difficult to discriminate between the exciton coupling within the dimer branches and those between the three branches.

The squared transition moments (see Table 1) of the squaraine absorption bands of the superchromophores show nearly additivity to their smaller analogues and thus follow the 


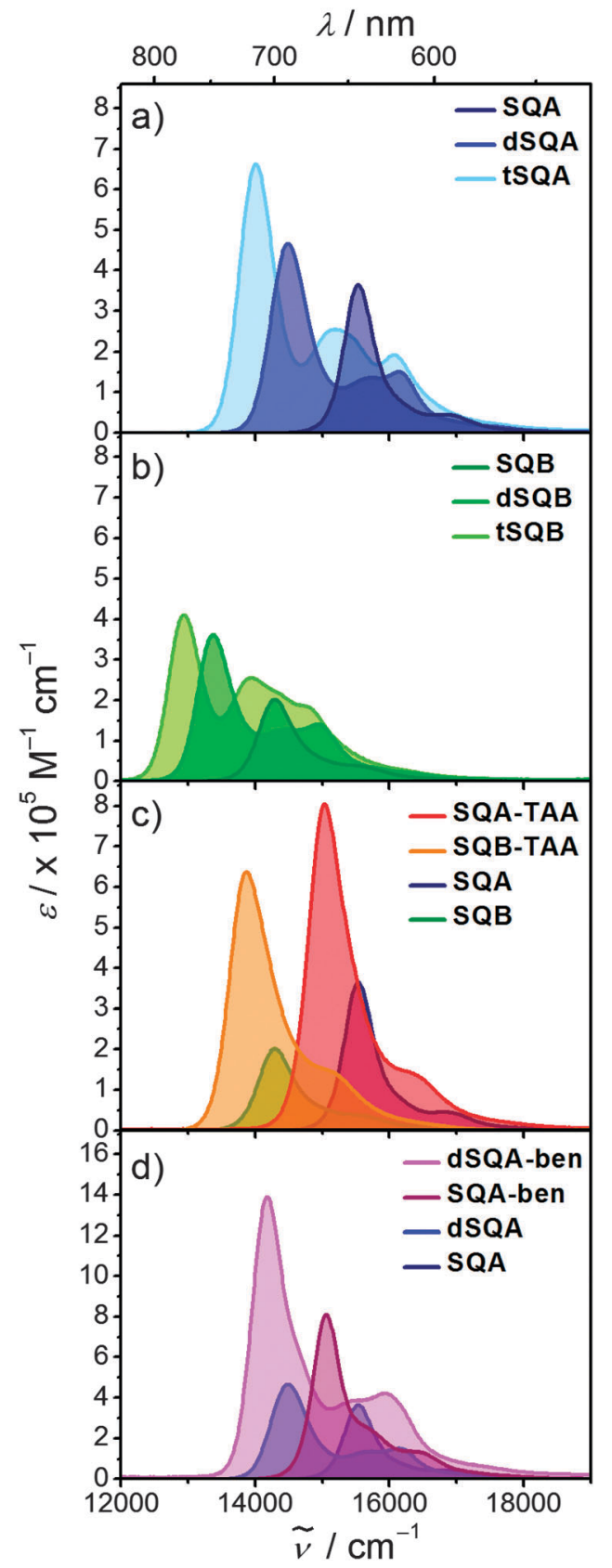

Fig. 3 1PA spectra of the investigated squaraines in toluene at rt.

Thomas-Reiche-Kuhn sum rule. ${ }^{42}$ This means, that no other electronic transitions with significant oscillator strength contribute to the exciton manifold. In all cases, two-exciton states ${ }^{43,44}$ can be anticipated as the result of the interaction of two excited chromophores (see Fig. 4). These two-exciton states cannot be reached by 1PA from the ground state but are visible as two consecutive absorption processes from the ground state to the 1PA state and from there to the two-exciton state in e.g. transient absorption spectroscopy experiments.

\section{Fluorescence spectra}

Upon excitation in the main absorption band the monomeric compounds SQA and SQB show typical squaraine fluorescence emission with a high quantum yield in toluene (Fig. 5). Their fluorescence spectra are the mirror image of the absorption with a small Stokes shift of $200-300 \mathrm{~cm}^{-1}$. The larger superchromophores all show similar behaviour, that is, the fluorescence looks similar to the one of the monomer but shifted to lower energies. Fluorescence lifetimes of all chromophores were measured by TCSPC in toluene. These lifetime measurements gave multiexponential decays in most cases (see Table 1). An alternative decay fit was done using the Kohlrausch equation (stretched exponential, eqn (3)) with exponents $\beta$ close to 1 which was equally good for most compounds. $^{45}$

$$
I=I_{0} \exp \left(-\left(t / \bar{\tau}_{\mathrm{f}}\right)^{\beta}\right)
$$

Only in some cases (dSQA, tSQA and dSQA-ben) a distribution analysis with logarithmically spaced lifetimes was necessary (see Table 1 and ESI $\dagger$ ). The reason for the fact that most squaraine dyes do not show monoexponential decays is unknown but might be caused by a distribution of conformers in case of the oligomers. While similar observations have been made by others before, ${ }^{46}$ this does not explain the multiexponential decays of e.g. SQA.

The average lifetimes acquired in this way were used to calculate the transition moment of the fluorescence $\mu_{\mathrm{f}}$ by the Strickler-Berg eqn $(4)^{47}$ from the fluorescence quantum yield $\phi_{\mathrm{f}}$.

$$
k_{\mathrm{f}}=\frac{16 \times 10^{6} \pi^{3}}{3 h \varepsilon_{0}} \frac{n\left(n^{2}+2\right)^{2}}{9}\left\langle\tilde{\nu}_{\mathrm{f}}{ }^{-3}\right\rangle_{\mathrm{av}}{ }^{-1} \mu_{\mathrm{f}}^{2}
$$

where $\left\langle\tilde{\nu}_{\mathrm{f}}{ }^{-3}\right\rangle_{\mathrm{av}}{ }^{-1}=\int I_{\mathrm{f}} \mathrm{d} \tilde{\nu} / \int \tilde{\nu}^{-3} I_{\mathrm{f}} \mathrm{d} \tilde{\nu}$ is the average cubic fluorescence energy and $k_{\mathrm{f}}$ is the radiative rate constant $k_{\mathrm{f}}=\phi_{\mathrm{f}} / \tau_{\mathrm{f}}$.

The squared fluorescence transition moments of SQA and SQB (see Table 1) are within the experimental uncertainty equal to those of the absorption. Thus, we discuss only relative squared transition moments. Here we observed a less than additive behaviour on going from SQA to dSQA and TSQA but still an increase which proves some microscopic superradiance effect, i.e. collective emission from delocalised states of chromophore aggregates. ${ }^{48-51}$ A similar effect was observed for dSQB and tSQB but not for all star-like superchromophores which indicates weak exciton coupling in the latter cases and emission from more localised states, that is, symmetry breaking takes place in the excited state. ${ }^{52,53}$

\section{Two photon absorption induced fluorescence}

The 2PAF spectra for the squaraine superchromophores in toluene are presented in Fig. 6 and the cross sections are given in Table 2. The measurement of 2PAF of tSQB was not possible because of the strongly red-shifted fluorescence which interfered with residual stray light from the $800 \mathrm{~nm}$ fundamental. Before we discuss the results of the superchromophores we will first present the 2PAF spectra of monomeric squaraines SQA and SQB and compare these results with those from the literature, where available.

For a centrosymmetric chromophore such as SQA the selection rules for electronic 1PA processes allow transitions from the ground state $\left(\mathrm{A}_{\mathrm{g}}\right)$ to the excited singlet state with $\mathrm{u}$ symmetry 


\section{a)}

two-exciton

states b)

c)

d)

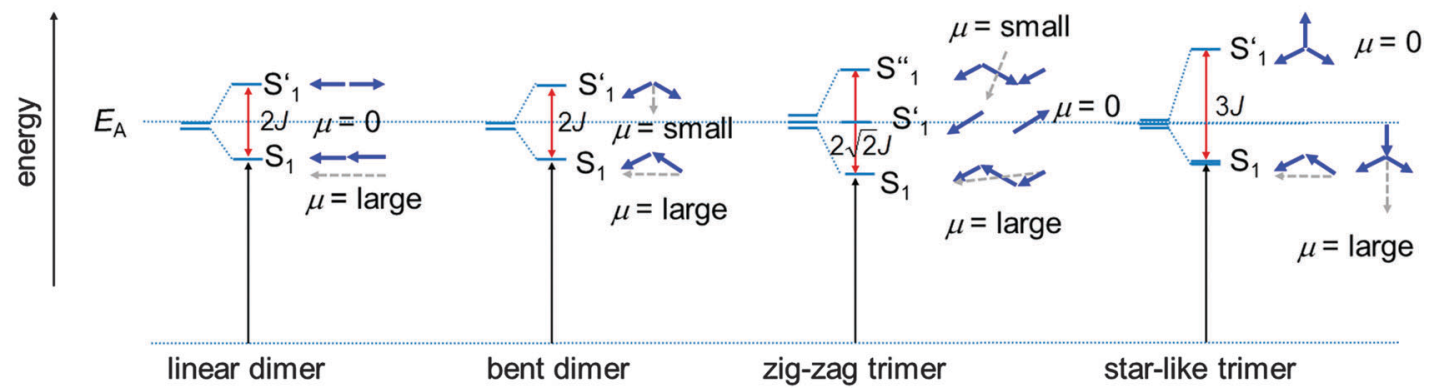

Fig. 4 Exciton eigenstates formed by exciton interaction in case of a linear dimer (a) and a bent dimer (b), a zig-zag trimer (c) and a star-like trimer (d). The blue arrows indicate the phase relations of the localised transition moments. Their length is proportional to their magnitude. The grey dashed arrows are the resulting sum of transition moments. Two-exciton states are formed by interaction of two excited chromophores.

Table 1 Optical data of the squaraine chromophores in toluene at $\mathrm{rt}$

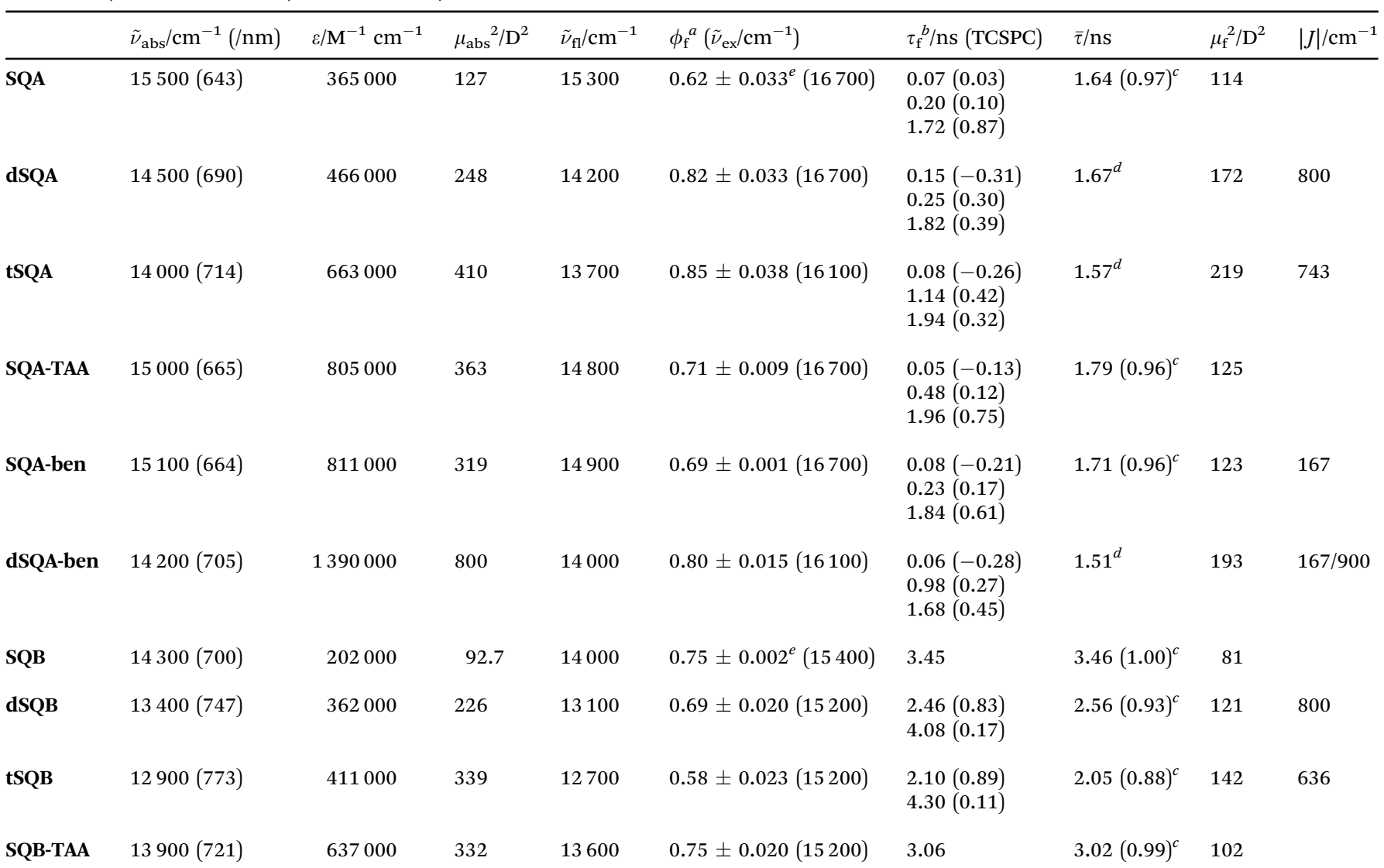

${ }^{a}$ Fluorescence quantum yield and excitation wavenumber used for measuring the fluorescence spectra in parentheses. The given error was determined by three independent measurements. ${ }^{b}$ Multiexponential fit of fluorescence decays measured by TSCPC, excitation at $15200 \mathrm{~cm}^{-1}$. Amplitudes are given in brackets. ${ }^{c}$ Lifetimes acquired by stretched exponential analysis of fluorescence decays measured by TCSPC, excitation at $15200 \mathrm{~cm}^{-1}$. Stretching exponent in brackets. ${ }^{d}$ Lifetimes acquired by distribution analysis of fluorescence decays measured by TCSPC, excitation at $15200 \mathrm{~cm}^{-1}$. The distribution is shown in Fig. S1 (ESI). ${ }^{e}$ This quantum yield deviates from older measurements ${ }^{39}$ because of the use of an improved set-up.

while the transitions to $\mathrm{g}$ symmetric states are dipole forbidden. ${ }^{54}$ Indeed, TD-DFT computations (see ESI $\dagger$ ) assign $B_{\mathrm{u}}$ symmetry to the lowest singlet excited state. According to this method the two next higher energy excitations are $\mathrm{n} \rightarrow \pi^{*}$ excitation of the oxygen lone pair electrons into the LUMO. Because of minimal orbital overlap these transitions possess vanishing oscillator strength 


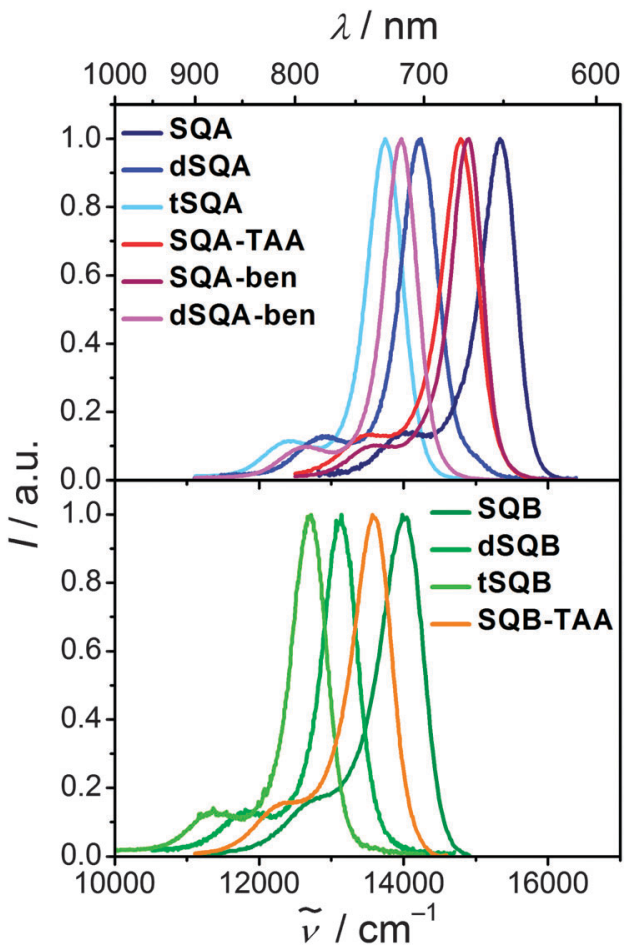

Fig. 5 Fluorescence spectra of squaraines dyes in toluene at rt.

and can neither be observed by 1PA nor by 2PA spectroscopy. This type of excitation is disregarded in the following. According to the computations the next orbital-overlap allowed state is an $\mathrm{A}_{\mathrm{g}}$ state which is 1PA forbidden but 2PA allowed. In our older work of SQA with $\mathrm{R}=$ ethyl in $\mathrm{CHCl}_{3}$, the symmetry of this state was confirmed by polarisation dependent $2 \mathrm{PAF}$ measurements. This state is at $24200 \mathrm{~cm}^{-1}$ and is also visible by fluorescence excitation anisotropy spectroscopy in viscous media. ${ }^{9}$ 2PAF spectroscopy also showed a weakly allowed transition at $16700 \mathrm{~cm}^{-1}$ just above the lowest $B_{\mathrm{u}}$ state at $15700 \mathrm{~cm}^{-1}$ (for SQA with $\mathrm{R}=$ ethyl in $\mathrm{CHCl}_{3}$ ). This transition is weakly 2PA allowed because of vibronic coupling to a $b_{\mathrm{u}}$ symmetric vibration $\left(B_{\mathrm{u}} \times b_{\mathrm{u}}=\mathrm{A}_{\mathrm{g}}\right) .{ }^{25}$ For SQA with $\mathrm{R}=$ dimethyloctyl in toluene we can confirm these older results (see Fig. 6) in our present work.

For SQB no 2PAF experiments are available in the literature, but, because of its $C_{2 \mathrm{v}}$ symmetry the 1PA and 2PA allowed states do not show the above mentioned parity selection rules. Thus, in principle all 1PA states are also 2PA allowed. Nevertheless, the 2PA spectrum of SQB is very similar to the one of SQA, showing a weak 2PA (100 GM) at the vibronic shoulder of the $S_{1}$ absorption at ca. $15700 \mathrm{~cm}^{-1}$ and a much higher $2 \mathrm{PA}$ of $\mathrm{S}_{2}$ at ca. $23000 \mathrm{~cm}^{-1}$. According to TD-DFT computations both states have $B_{2}$ symmetry and are polarised along the long axis of the molecule. $\mathrm{S}_{3}$ is an $\mathrm{A}_{1}$ state and polarised along the molecular $\mathrm{C}_{2}$ axis but is out of reach for the 2PA experiments. This agrees very well with the fluorescence excitation anisotropy (FEA) spectrum (see $\mathrm{ESI}^{\dagger}$ ).

The 2PAF spectrum of the dimer dSQA shows dramatic differences in comparison to SQA: in addition to the vibronically allowed 2PA peak at the shoulder of the $\mathrm{S}_{1} \leftarrow \mathrm{S}_{0}$ transition
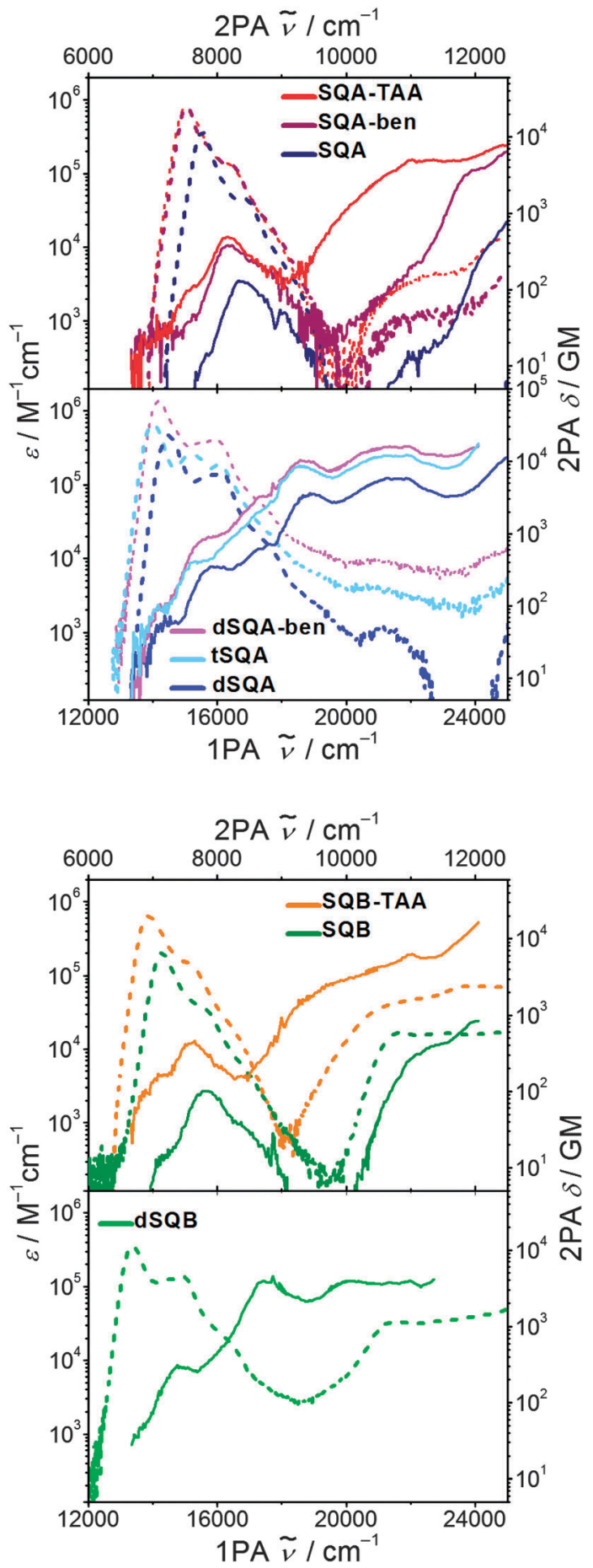

Fig. 6 Two photon absorption spectra (solid lines) and one photon absorption spectra (dashed lines) of the investigated squaraines in toluene at rt.

at $14500 \mathrm{~cm}^{-155}$ there are pronounced 2PA maxima at ca. $18800 \mathrm{~cm}^{-1}\left(\delta_{2 \mathrm{PA}}=3430 \mathrm{GM}\right)$ and at $21800 \mathrm{~cm}^{-1}$ $\left(\delta_{2 \mathrm{PA}}=5780 \mathrm{GM}\right)$ which are absent in the monomer SQA. A comparison of the 1PA and the 2PA spectra on a logarithmic scale together with the fluorescence excitation anisotropy (FEA) spectrum allows a more detailed assignment of states (see Fig. 7). The FEA value $r=0.36$ around the $0-0$ peak of the $S_{1}$ state proves 
Table 2 One photon cross section at the absorption maximum and two photon cross sections at the maxima of the squaraines in toluene at rt ${ }^{b}$

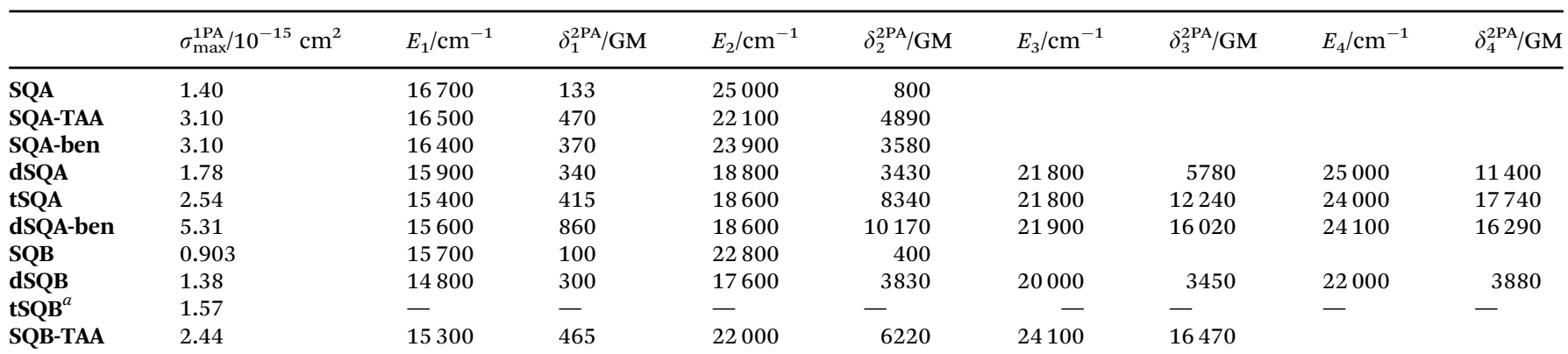

${ }^{a}$ Could not be measured because of red-shifted fluorescence. ${ }^{b}$ The fact that the $2 \mathrm{PA}$ cross sections are grouped in columns does not necessarily mean that they derive from similar electronic states.

this transition to be polarised parallel to the emission transition moment (ideally $r=0.4$ in this case). ${ }^{56}$ The FEA drops to ca. 0.2 at the $\mathrm{S}_{1}(0-1)$ transition and finally to $c a .-0.03$ at the $\mathrm{S}_{1}{ }^{\prime}$ state and to $c a .0 .09$ for its associated 0-1 transition. The latter values indicate a pronounced angle between the transition moments of these excitations and that of the fluorescence $\left(S_{1} \rightarrow S_{0}\right)$. Indeed, for the bent dimer case Fig. $4 \mathrm{~b}$ shows a perpendicular orientation of the $\mathrm{S}_{1}{ }^{\prime} \leftarrow \mathrm{S}_{0}$ transition moment which would ideally give $r=-0.2$. The following states (termed $\mathrm{S}_{1}{ }^{\prime \prime}$ and $\left.\mathrm{S}_{1}{ }^{\prime \prime \prime}\right)$ are visible in the 2PA spectra as pronounced peaks, in the FEA as maxima $(r=0.2)$ and minima $(r=0.07)$ but also as weak shoulders and peaks in the 1PA spectrum at ca. $18800 \mathrm{~cm}^{-1}$ and at $21400 \mathrm{~cm}^{-1}$. While the $S_{1}$ and $S_{1}{ }^{\prime}$ states can easily be derived by exciton coupling theory, $\mathrm{S}_{1}{ }^{\prime \prime}$ and $\mathrm{S}_{1}{ }^{\prime \prime \prime}$ cannot. Therefore, assuming a linear dimer situation we construct an orbital interaction diagram as given in the $\mathrm{ESI}^{\dagger} \dagger$ in which the $\pi$-HOMO and the $\pi$-LUMO of a monomer interact and give in total four new dimer $\pi$-orbitals. With these four orbitals, four singly excited configurations can be generated. Because of their orbital symmetry (either $\mathrm{g}$ or $\mathrm{u}$ ) this gives rise to two allowed and two forbidden transitions from the ground state. ${ }^{57}$ TD-DFT computations come to the same conclusion (see ESI $\dagger$ ). ${ }^{58}$ Thus, four exited states may be derived from the orbital interaction of two monomers while simple excited state

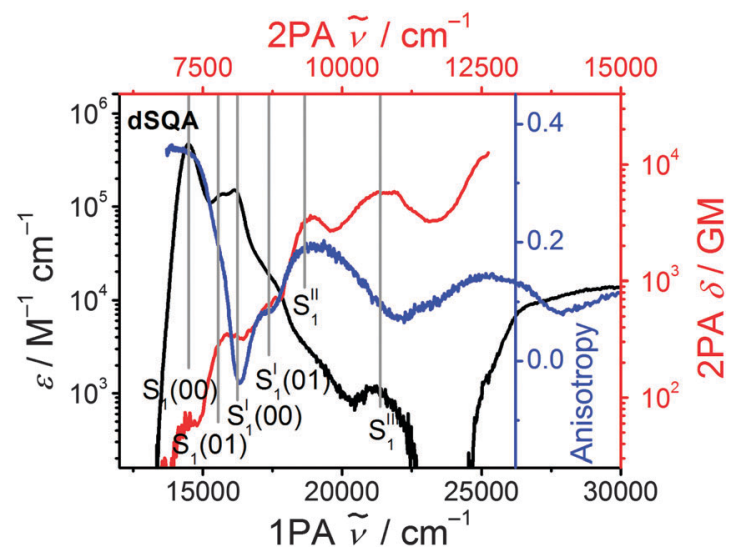

Fig. 7 1PA (black) and the 2PA (red) spectra of dSQA in toluene on a logarithmic scale together with the fluorescence excitation anisotropy (FEA) spectrum (blue) in polyTHF at $26^{\circ} \mathrm{C}$. exciton interaction theory predicts only two states. Therefore, it appears that exciton coupling theory is intrinsically insufficient to cover all aspects of 2PA properties. We assume that the four electronic states that we observe experimentally up to $23000 \mathrm{~cm}^{-1}$ are the four states that we constructed from orbital mixing. Because these states are closely related to $S_{1}$ of the monomer they are consequently numbered $\mathrm{S}_{1}$ through $\mathrm{S}_{1}{ }^{\prime \prime}$. The relative order of these states is difficult to assign as CI mixing may lead to energy shifts of the simple MO configurations and, furthermore, intensity borrowing between states of same symmetry may alter the observed intensities. ${ }^{59-61}$

In the trimer tSQA the 1PA and 2PA allowed states are shifted a little bit towards lower energy (see Fig. 6 and Table 2). More interesting is that the 2PA cross section for the $\mathrm{S}_{1}{ }^{\prime \prime}$ and $\mathrm{S}_{1}{ }^{\prime \prime \prime}$ band is about twice that of the dimer (at energies $E_{2}$ and $E_{3}$, see Table 2), thus showing a significant cooperative enhancement (see eqn (2)) of $\delta_{2 \mathrm{PA}}$ at very similar state energies. Beyond these general statements it is difficult to draw more specific conclusions about possible $n^{2}$ dependencies of the 2PA cross sections. This has to do with the fact that one can only compare 2PA cross sections which derive from similar electronic states, this is possible for e.g. the $\mathrm{S}_{1}{ }^{\prime \prime}$ and $\mathrm{S}_{1}{ }^{\prime \prime \prime}$ states of ISQA and tSQA but not for SQA where these states are missing. For the former, a relative $2 \mathrm{PA}$ cross section of $4: 9.7$ is obtained for $\mathrm{S}_{1}{ }^{\prime \prime}$ at $18800 \mathrm{~cm}^{-1}$ and of $4: 8.5$ for $\mathrm{S}_{1}{ }^{\prime \prime \prime}$ at $21800 \mathrm{~cm}^{-1}$. Both these ratios are very close to the $4: 9$ ratio expected for a dimer and a trimer.

The dimer dSQB shows a surprisingly similar 2PA spectrum as ISQA with the individual peaks a little bit shifted to lower energies (Fig. 8). The 2PA cross sections are also very similar. This demonstrates that the principal components of the 1PA and 2PA allowed states are in both dimers formed by interaction of the transitions polarised along the molecular long axis and that those polarised perpendicular to the long axis do not play a significant role. ${ }^{62}$

The star-like superchromophores SQA-TAA and SQA-ben display qualitatively similar 2PA spectra as SQA. In particular there is a pronounced 2PA at the energy of the vibronic progression at $c a .17000 \mathrm{~cm}^{-1}$ (see Table 2). Because of three squaraine chromophores in the star-like systems the 2PA is about three times that of SQA in this energy region. The 2PA at higher energy $\left(>22000 \mathrm{~cm}^{-1}\right)$ also rises for the star-like superchromophores, 


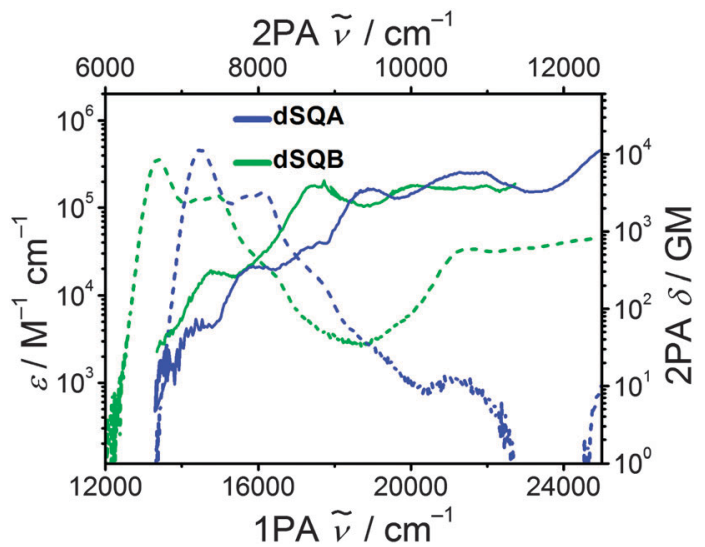

Fig. 8 Two photon absorption spectra (solid lines) and one photon absorption spectra (dashed lines) of the dimers dSQA and dSQB in toluene at rt.

in particular for SQA-TAA. This effect cannot be caused by a double resonance effect with the 1PA state because the lowest 1PA energy is almost the same in SQA, SQA-ben and SQA-TAA. We suppose that it is caused by an increase of density of states because of the enlargement of the $\pi$-system, possibly also because of states with CT character in the case of SQA-TAA. This effect is even stronger in the case of SQB-TAA.

Much in contrast, the 2PA of dSQA-ben at the $\mathrm{S}_{1}$ to $\mathrm{S}_{1}{ }^{\prime \prime}$ energies is almost exactly three times that of the corresponding 2PA values of dSQA thus showing additivity of the 2PA cross section. Consequently, while there is a cooperative enhancement extending ISQA by one squaraine chromophore to tSQA there is additivity on going from dSQA to dSQA-ben. This demonstrates that the interactions between the branches of the 1,3,5-substituted benzene are negligible but they are strong in the linear arrangement of squaraine dyes.

However, the fluorescence quantum yield is one of the major sources of error in the determination of the 2PA cross section. ${ }^{63}$ Particularly in case of SQA-ben and dSQA-ben the quantum yields may possess a systematic error because of surface adsorption effects to the cuvette walls. Therefore, the conclusions concerning those chromophores should be taken with care.

For all squaraine superchromophores the 2PA cross section rises strongly when the excitation energy approaches the 1PA energy. This is, as mentioned in the introduction, the consequence of a double resonance effect because for all exciton coupled chromophores, there are biexciton states at about twice the energy of the 1PA states (see Fig. 4). Although the biexciton states cannot simply be characterised as such by 2PA spectroscopy, they may have an indirect influence via the double resonance effect.

\section{Conclusions}

In conclusion, all investigated squaraines and squaraine superchromophores displayed sizeable 2PA cross sections. The spectral regions where 2PA resonances occur can be divided into several sections (from low to high energies): (1) for all squaraines the vibronic shoulder at the 1PA energy is 2PA allowed because of vibronic coupling. (2) In the superchromophores dSQA, tSQA, dSQA-ben and dSQB, there is strong 2PA at higher energy states within the exciton manifold. This is caused by symmetry breaking in the superchromophores which lifts the parity rule. (3) For the same superchromophores as above there are low-lying 2PA states which may be understood as being formed by orbital interactions in the linear sequence of squaraine dyes. (4) In case of squaraines with a triarylamine core, the intermediate 2PA allowed states shift to lower energies possibly because of CT character. (5) For all squaraines there is a strong 2PA approaching twice the energy of the 1PA states because of double resonance condition.

Besides these findings we found enhancement of 2PA cross sections in linear arrangements of squaraines (dSQA, tSQA, dSQB) but not in those with star-like arrangement (SQA-ben, dSQA-ben) where merely additivity of 2PA cross section is found. The latter is in accord with observations made by Blanchard-Desce et $a l^{22}$ for other superchromophores with a benzene core. This is surprising given the high oscillator strength of the squaraine dyes. This should lead to a sizable Coulomb coupling between squaraine chromophores even though they are connected by a benzene ring in 1,3,5-position as is evident by comparing the 1PA spectra of SQA and SQA-ben where moderate excitonic couplings were found. Obviously, this is not the case for higher excited states and only additivity of 2PA cross section is therefore observed. Similarly, we found no superradiance effect for branched chromophores but for all linear arrangements. All these observation suggest that the enhancement of 2PA cross section in the linear arrangements of ASQA and tSQA is probably caused by orbital interactions of higher excited configurations.

\section{Experimental}

\section{Steady-state absorption spectroscopy}

All dyes were dissolved in toluene (Uvasol from Merck) and the $\mathrm{UV} / \mathrm{vis} / \mathrm{NIR}$-absorption spectra were measured in $1 \mathrm{~cm}$ quartz cuvettes from Hellma using a Jasco V-670 spectrometer. The pure solvent was used as reference.

The squares of the transition dipole moment were obtained by integration of the main low energy absorption band and calculated as follows ${ }^{64}$

$$
\mu_{\mathrm{eg}}^{2}=\frac{3 h c \varepsilon_{0} \ln 10}{2000 \pi^{2} N_{\mathrm{AV}}} \frac{9 n}{\left(n^{2}+2\right)^{2}} \int \frac{\varepsilon}{\tilde{\nu}} \mathrm{d} \tilde{\nu}
$$

\section{Emission spectroscopy}

Steady-state fluorescence measurements were performed with an Edinburgh Instruments FLS980 spectrometer. The compounds were dissolved in toluene (Uvasol from Merck) and purged with argon gas for $30 \mathrm{~min}$ prior to each measurement. Fluorescence quantum yields were measured with an integrating sphere and the FLS980 spectrometer applying the method of Bardeen et al. ${ }^{65}$ to correct for self-absorption. Fluorescence lifetimes were determined by time-correlated single-photon counting (TCSPC) with the FLS980 spectrometer by exciting the samples with a pulsed laser 
diode at $15200 \mathrm{~cm}^{-1}$ (656 nm) under magic angle conditions and using a fast PMT detector (H10720) for fluorescence detection. Deconvolution of the data (4096 channels) was done by measuring the instrument response function with a scatterer (LUDOX). For the fluorescence anisotropy measurement the fluorimeter was equipped with two polarisation filters. The compounds were dissolved in polyTHF $\left(M_{\mathrm{n}} \sim 650\right.$, CAS 25190-06-1, Sigma Aldrich) and measured at $26{ }^{\circ} \mathrm{C}$ and $20{ }^{\circ} \mathrm{C}$.

\section{Two photon absorption setup}

Two-photon cross sections were determined via two-photon excitation spectra using a set-up similar to the one described in ref. 55. In detail, the output of a TOPAS-Prime in combination with a NirUVis frequency mixer (both from Light Conversion) was used as excitation source. The excitation intensity was adjusted using a combination of a broadband zero-order halfwaveplate and a Glan-Taylor polarizer, and the polarization set to vertical. The beam was slightly focused by a $(f=20 \mathrm{~cm})$ lens, which was placed $10 \mathrm{~cm}$ before the sample. The pump power was monitored using a powermeter (Thorlabs PM100A) equipped with a thermal sensor (Thorlabs S302C) behind the sample. The fluorescence was focused onto the entrance slit of a monochromator $(0.25 \mathrm{~m}$ Cornerstone, Oriel, grating 74166 Newport) equipped with a multi-pixel photon-counter avalanche photodiode detector (Hamamatsu S-10362-11-050U) using a spherical mirror ( $\varnothing=75 \mathrm{~mm}, f=150 \mathrm{~mm}$ ). The output signal was preamplified (SR240, Stanford Research Systems), processed with a gated boxcar-integrator and averager module (SR250, SRS), digitized (SR245, SRS) and recorded on a computer.

The two photon cross section at a given wavenumber, $\delta_{\mathrm{s}}^{(2)}(\tilde{\nu})$, was calculated as follows ${ }^{66}$

$$
\delta_{\mathrm{s}}^{(2)}(\tilde{\nu})=\frac{I_{\mathrm{s}}\left(\tilde{\nu}, \lambda_{\text {obs }}\right) c_{\mathrm{r}} \phi_{\mathrm{r}}\left(\lambda_{\text {obs }}\right)}{I_{\mathrm{r}}\left(\tilde{\nu}, \lambda_{\text {obs }}\right) c_{\mathrm{s}} \phi_{\mathrm{s}}\left(\lambda_{\mathrm{obs}}\right)} \delta_{\mathrm{r}}^{(2)}(\tilde{\nu})
$$

Here $I_{x}\left(\tilde{\nu}, \lambda_{\text {obs }}\right)$ is the (two-photon induced) fluorescence intensity at excitation wavenumber $\nu$ and observation wavelength $\lambda_{\text {obs }}$ for either sample or reference $(x \in\{\mathrm{s}, \mathrm{r}\}) . c_{x}$ and $\phi_{x}\left(\lambda_{\text {obs }}\right)$ are the concentration and differential fluorescence quantum yield (at the observation wavelength) of sample and reference. LDS698 and Styryl $9 \mathrm{M}$ in chloroform were used as ref. 66 and 67.

The power dependence of the fluorescence intensity was monitored throughout the measurement (Fig. S3, ESI $\dagger$ ).

\section{Acknowledgements}

The Würzburg group thanks the Deutsche Forschungsgemeinschaft for financial support of this work within the Research Group FOR1809. The Geneva group wish to thank the Fonds National Suisse de la Recherche Scientifique through project no. 200020-165890.

\section{Notes and references}

1 S. J. Chung, S. J. Zheng, T. Odani, L. Beverina, J. Fu, L. A. Padilha, A. Biesso, J. M. Hales, X. W. Zhan, K. Schmidt, A. J. Ye, E. Zojer, S. Barlow, D. J. Hagan, E. W. Van Stryland,
Y. P. Yi, Z. G. Shuai, G. A. Pagani, J. L. Bredas, J. W. Perry and S. R. Marder, J. Am. Chem. Soc., 2006, 128, 14444-14445.

2 B. Strehmel, S. Amthor, J. Schelter and C. Lambert, ChemPhysChem, 2005, 6, 893-896.

3 W. Denk, J. H. Strickler and W. W. Webb, Science, 1990, 248, 73-76.

4 C. Xu and W. W. Webb, J. Opt. Soc. Am. B, 1996, 13, 481-491.

5 D. A. Parthenopoulos and P. M. Rentzepis, Science, 1989, 245, 843-845.

6 J. D. Bhawalkar, N. D. Kumar, C. F. Zhao and P. N. Prasad, Journal of clinical laser medicine \& surgery, 1997, 15, 201-204.

7 S. J. Andrasik, K. D. Belfield, M. V. Bondar, F. E. Hernandez, A. R. Morales, O. V. Przhonska and S. Yao, ChemPhysChem, 2007, 8, 399-404.

8 K. Kamada, K. Ohta, I. Yoichiro and K. Kondo, Chem. Phys. Lett., 2003, 372, 386-393.

9 J. Fu, L. A. Padilha, D. J. Hagan, E. W. Van Stryland, O. V. Przhonska, M. V. Bondar, Y. L. Slominsky and A. D. Kachkovski, J. Opt. Soc. Am. B, 2007, 24, 67-76.

10 M. Drobizhev, A. Karotki, M. Kruk and A. Rebane, Chem. Phys. Lett., 2002, 355, 175-182.

11 M. Albota, D. Beljonne, J. L. Bredas, J. E. Ehrlich, J. Y. Fu, A. A. Heikal, S. E. Hess, T. Kogej, M. D. Levin, S. R. Marder, D. McCord-Maughon, J. W. Perry, H. Rockel, M. Rumi, C. Subramaniam, W. W. Webb, X. L. Wu and C. Xu, Science, 1998, 281, 1653-1656.

12 M. Rumi, J. E. Ehrlich, A. A. Heikal, J. W. Perry, S. Barlow, Z. Y. Hu, D. McCord-Maughon, T. C. Parker, H. Rockel, S. Thayumanavan, S. R. Marder, D. Beljonne and J. L. Bredas, J. Am. Chem. Soc., 2000, 122, 9500-9510.

13 S. Webster, J. Fu, L. A. Padilha, O. V. Przhonska, D. J. Hagan, E. W. Van Stryland, M. V. Bondar, Y. L. Slominsky and A. D. Kachkovski, Chem. Phys., 2008, 348, 143-151.

14 S. Webster, S. A. Odom, L. A. Padilha, O. V. Przhonska, D. Peceli, H. H. Hu, G. Nootz, A. D. Kachkovski, J. Matichak, S. Barlow, H. L. Anderson, S. R. Marder, D. J. Hagan and E. W. Van Stryland, J. Phys. Chem. B, 2009, 113, 14854-14867. 15 W. V. Moreshead, O. V. Przhonska, M. V. Bondar, A. D. Kachkovski, I. H. Nayyar, A. E. Masunov, A. W. Woodward and K. D. Belfield, J. Phys. Chem. C, 2013, 117, 23133-23147.

16 C. Andraud, R. Fortrie, C. Barsu, O. Stéphan, H. Chermette and P. L. Baldeck, in Photoresponsive Polymers II, ed. S. R. Marder and K.-S. Lee, Springer, Berlin, Heidelberg, 2008, vol. 214.

17 F. Terenziani, C. Katan, E. Badaeva, S. Tretiak and M. BlanchardDesce, Adv. Mater., 2008, 20, 4641-4678.

18 E. Collini, Phys. Chem. Chem. Phys., 2012, 14, 3725-3736.

19 M. G. Kuzyk, J. Chem. Phys., 2003, 119, 8327-8334.

20 M. Drobizhev, A. Karotki, Y. Dzenis, A. Rebane, Z. Suo and C. W. Spangler, J. Phys. Chem. B, 2003, 107, 7540-7543.

21 C. Katan, F. Terenziani, O. Mongin, M. H. V. Werts, L. Porres, T. Pons, J. Mertz, S. Tretiak and M. Blanchard-Desce, J. Phys. Chem. A, 2005, 109, 3024-3037.

22 F. Terenziani, C. Le Droumaguet, C. Katan, O. Mongin and M. Blanchard-Desce, ChemPhysChem, 2007, 8, 723-734. 
23 D. Beljonne, W. Wenseleers, E. Zojer, Z. G. Shuai, H. Vogel, S. J. K. Pond, J. W. Perry, S. R. Marder and J. L. Bredas, Adv. Funct. Mater., 2002, 12, 631-641.

24 S. J. Chung, K. S. Kim, T. H. Lin, G. S. He, J. Swiatkiewicz and P. N. Prasad, J. Phys. Chem. B, 1999, 103, 10741-10745.

25 D. Scherer, R. Dorfler, A. Feldner, T. Vogtmann, M. Schwoerer, U. Lawrentz, W. Grahn and C. Lambert, Chem. Phys., 2002, 279, 179-207.

26 L. Beverina and M. Sassi, Synlett, 2014, 477-490.

27 L. Beverina and P. Salice, Eur. J. Org. Chem., 2010, 1207-1225.

28 A. Ajayaghosh, Chem. Soc. Rev., 2003, 32, 181-191.

29 A. Ajayaghosh, Acc. Chem. Res., 2005, 38, 449-459.

30 S. Sreejith, P. Carol, P. Chithra and A. Ajayaghosh, J. Mater. Chem., 2008, 18, 264-274.

31 P. Anees, S. Sreejith and A. Ajayaghosh, J. Am. Chem. Soc., 2014, 136, 13233-13239.

32 T. Geiger, S. Kuster, J. H. Yum, S. J. Moon, M. K. Nazeeruddin, M. Gratzel and F. Nuesch, Adv. Funct. Mater., 2009, 19, 2720-2727.

33 S. Kuster and T. Geiger, Dyes Pigm., 2012, 95, 657-670.

34 L. Hu, Z. Q. Yan and H. Y. Xu, RSC Adv., 2013, 3, 7667-7676.

35 S. A. Odom, S. Webster, L. A. Padilha, D. Peceli, H. Hu, G. Nootz, S. J. Chung, S. Ohira, J. D. Matichak, O. V. Przhonska, A. D. Kachkovski, S. Barlow, J. L. Bredas, H. L. Anderson, D. J. Hagan, E. W. Van Stryland and S. R. Marder, J. Am. Chem. Soc., 2009, 131, 7510-7511.

36 S. Ohira, I. Rudra, K. Schmidt, S. Barlow, S. J. Chung, Q. Zhang, J. Matichak, S. R. Marder and J. L. Bredas, Chem. - Eur. J., 2008, 14, 11082-11091.

37 C. Lambert, T. Scherpf, H. Ceymann, A. Schmiedel and M. Holzapfel, J. Am. Chem. Soc., 2015, 137, 3547-3557.

38 M. Kasha, H. R. Rawls and M. A. El-Bayoumi, Pure Appl. Chem., 1965, 11, 371-392.

39 S. F. Völker, A. Schmiedel, M. Holzapfel, K. Renziehausen, V. Engel and C. Lambert, J. Phys. Chem. C, 2014, 118, 17467-17482.

40 S. F. Völker, T. Dellermann, H. Ceymann, M. Holzapfel and C. Lambert, J. Polym. Sci., Part A: Polym. Chem., 2014, 52, 890-911.

41 S. F. Völker, M. Renz, M. Kaupp and C. Lambert, Chem. - Eur. J., 2011, 17, 14147-14163.

42 W. W. Parson, Modern Optical Spectroscopy, Springer, 2009, p. 293.

43 H. v. Amerongen, L. Valkunas and R. v. Grondelle, Photosynthetic Excitons, World Scientific, Singapur, 2002, ch. 3, pp. 73-118.

44 J. Knoester, in Proceedings of the International School of Physics "Enrico Fermi" Course CXLIX, ed. V. M. Agranovich and G. C. La Rocca, IOS Press, Amsterdam, 2002, pp. 149-186.

45 A recently performed fluorescence upconversion measurement of SQA in toluene at $590 \mathrm{~nm}$ pump [see ref. 37] was reanalysed using a stretched exponential function and gave $\tau_{\mathrm{f}}=1.57 \mathrm{~ns}$ and $\beta=0.90$.
46 G. de Miguel, M. Marchena, M. Zitnan, S. S. Pandey, S. Hayase and A. Douhal, Phys. Chem. Chem. Phys., 2012, 14, 1796-1805.

47 S. J. Strickler and R. A. Berg, J. Chem. Phys., 1962, 37, 814-822.

48 R. H. Dicke, Phys. Rev., 1954, 93, 99-110.

49 G. L. Celardo, G. G. Giusteri and F. Borgonovi, Phys. Rev. B: Condens. Matter Mater. Phys., 2014, 90, 075113.

50 H. Fidder, J. Knoester and D. A. Wiersma, J. Chem. Phys., 1991, 95, 7880-7890.

51 We stress that the use of the term "superradiance" in this work differs from the older definition of a "macroscopic superradiance” by Dicke, R. H. Dicke, Phys. Rev., 1954, 93, 99-110see also F. C. Spano and S. Mukamel, J. Chem. Phys., 1989, 91, 683-700.

52 E. Vauthey, ChemPhysChem, 2012, 13, 2001-2011.

53 H. Ceymann, M. Balkenhohl, A. Schmiedel, M. Holzapfel and C. Lambert, Phys. Chem. Chem. Phys., 2016, 18, 2646-2657.

54 M. A. C. Nascimento, Chem. Phys., 1983, 74, 51-66.

55 We refrain from discussing the relative intensities of these vibronically allowed 2PA bands of the diverse squaraine dyes as intensity borrowing may blur any interpretation in terms of cooperative effects.

56 J. R. Lakowicz, Principles of Fluorescence Spectroscopy, Springer, New York, NY, 3. (corr. at 4. print.) edn, 2010.

57 The two $g$ symmetric configurations maybe undergo CI mixing.

58 For a comparison of different methods to estimate exciton coupling effects, see W. J. D. Beenken and T. Pullerits, J. Chem. Phys., 2004, 120, 2490-2495; W. L. Liu, V. Settels, P. H. P. Harbach, A. Dreuw, R. F. Fink and B. Engels, J. Comput. Chem., 2011, 32, 1971-1981.

59 Y. Shibata, S. Tateishi, S. Nakabayashi, S. Itoh and H. Tamiaki, Biochemistry, 2010, 49, 7504-7515.

60 D. Gülen, Photosynth. Res., 2006, 87, 205-214.

61 P. Macak, Y. Luo, P. Norman and H. Ågren, J. Chem. Phys., 2000, 113, 7055-7061.

62 Bredas et al. recently pointed out that non-centrosymmetrical cis-squaraines behave essentially as centrosymmetrical trans-squaraines concerning the properties of excited states [see ref. 36].

63 Due to the unknown systematic errors of the fluorescence quantum yields we refrain from giving errors for the $2 \mathrm{PA}$ measurements, but we estimate those being within $10 \%$ relative to each other for the set of compounds studied here.

64 J. E. Lewis and M. Maroncelli, Chem. Phys. Lett., 1998, 282, 197-203.

65 T. S. Ahn, R. O. Al-Kaysi, A. M. Mueller, K. M. Wentz and C. J. Bardeen, Rev. Sci. Instrum., 2007, 78, 086105.

66 N. S. Makarov, M. Drobizhev and A. Rebane, Opt. Express, 2008, 16, 4029-4047.

67 N. S. Makarov, J. Campo, J. M. Hales and J. W. Perry, Opt. Mater. Express, 2011, 1, 551-563. 